\title{
Human platelet lysate-based nanocomposite bioink for bioprinting hierarchical fibrillar structures
}

\author{
Bárbara B. Mendes $\dagger^{1,2,3}$, Manuel Gómez-Florit ${ }^{\dagger^{1,2,3}}$, Alex G. Hamilton ${ }^{4}$, Michael S. \\ Detamore $^{4}$, Rui M. A. Domingues ${ }^{1,2,3 *}$ Rui L. Reis ${ }^{1,2,3}$ and Manuela E. Gomes ${ }^{1,2,3 *}$ \\ 1 3B's Research Group, I3Bs - Research Institute on Biomaterials, Biodegradables and Biomimetics, \\ University of Minho, Headquarters of the European Institute of Excellence on Tissue Engineering and \\ Regenerative Medicine, Avepark, Zona Industrial da Gandra, 4805-017 Barco - Guimarães, Portugal. \\ 2 ICVS/3B's - PT Government Associate Laboratory, Braga/Guimarães 4805-017, Portugal. \\ ${ }^{3}$ The Discoveries Centre for Regenerative and Precision Medicine, Headquarters at University of Minho, \\ Avepark, 4805-017 Barco, Guimarães, Portugal. \\ ${ }^{4}$ Stephenson School of Biomedical Engineering, The University of Oklahoma, Norman, OK, USA. \\ $\uparrow$ Both authors contributed equally to this work.
}

E-mail: megomes@i3bs.uminho.pt, rui.domingues@i3bs.uminho.pt

Received xxxxxx

Accepted for publication $\mathrm{xxxxxx}$

Published xxxxxx

\begin{abstract}
Three-dimensional (3D) bioprinting holds the promise to fabricate tissue and organ substitutes for regenerative medicine. However, the lack of bioactive inks to fabricate and support functional living constructs is one of the main limitations hindering the progress of this technology. In this study, a biofunctional human-based nanocomposite bioink (HUink) composed of platelet lysate hydrogels reinforced by cellulose nanocrystals is reported. When combined with suspended bioprinting technologies, HUink allows the biofabrication of 3D freeform constructs with high resolution and integrity, mimicking the hierarchical nano-tomacro fibrillary composition of native tissues. Remarkably, HUink supports bioprinting of stem cells with high viability immediately after extrusion and over long-term cell culture without the need for additional biochemical or animal-derived media supplementation. As opposed to typical polymer-based bioinks, the pool of growth factors, cytokines and adhesion proteins in HUink boosts cell spreading and proliferation, stimulating the fast production of cell-secreted extracellular matrix. This innovative bioprinting platform with unpaired biofunctionality allows the fabrication of complex freeform cell-laden constructs that can ultimately be applied in the development of xeno-free 3D tissue models for in vitro research or to develop tissue and organ surrogates for clinical applications.
\end{abstract}

Keywords: bioprinting, platelet lysate, bioinks, bioactive, xeno-free

Supplementary material (SM) for this article is available online

\section{Introduction}

Three-dimensional (3D) bioprinting technology, in particular extrusion-based systems, have been receiving considerable attention in tissue engineering and regenerative medicine as a cutting-edge technique to fabricate functional tissue and organ surrogates. $[1,2]$ However, commonly used bioinks, i.e. biomaterials that contain biological molecules 
and/or cells, are often limited to the "biofabrication window", in which the proposed hydrogel inks cannot be printed with high resolution and under cytocompatible conditions, delaying the clinical translation of personalized bioprinted structures.[3, 4] The main limitations of traditional hydrogelbased bioinks are generally related to the high polymer concentration and crosslinking density needed for the biofabrication of constructs with high printing fidelity, which compete with their biocompatibility and leads to detrimental effects over cell survival, spreading and nutrient diffusion.[3, 5] In order to overcome these limitations, current research has focused on the development of advanced bioinks based on supramolecular, multimaterial and nanocomposite hydrogels, a topic which has been widely covered by recent reviews.[2, 3] Another key aspect of advanced bioinks is their biofunctionality, as they should ideally be able to build or recreate the organization of extracellular matrix (ECM) in the native tissues and its role in supporting the cell survival, organization and differentiation of embedded cells. The functionalization of hydrogel polymers with cell-adhesion peptide sequences, stimuli responsive moieties or incorporation/conjugation of growth factors has been explored to improve the biological properties of bioinks.[6] Even though, the multiple synthesis steps required by such strategies as well as the need to use high doses of recombinant growth factors, due to their fast degradation and loss of bioactivity, represents prohibitive costs for widespread application.[7] Another important and generally overlooked limitation of hydrogels is that they are formed from crosslinked networks of polymer molecules which do not mimic the fibrous nature of the ECM. The filamentous architecture of the ECM strongly influences normal cell and organ function because it has a fundamental impact over e.g. cells' phenotype, migration, mechanotransduction, growth factors signalling pathways and mass transport of nutrients and waste (due to the large pore size of their networks)[8-10]. To recreate the native ECM fibre-based architecture and topology, different bioinks have been developed based on decellularised ECM [11] or the self-assembly of biomolecules, e.g. peptide amphiphiles, into biomimetic 3D fibrillar hydrogels.[12, 13] However, decellularised ECM hydrogels are typically derived from animal tissues and show high variability, while the gelation mechanisms of selfassembled fibrillar hydrogels usually proceed in nonbiocompatible conditions that together with intricate synthesis pathways and lack of intrinsic biofunctionality, still limits the potential of these biomaterials in this field. Overall, most of the current bioinks are not yet able to mimic the native tissue's ECM fibrillar architecture across multiple length scales while simultaneously showing high biocompatibility and incorporating key cell instructive bioactive elements, which are critical to move 3D bioprinting a step towards the generation of functional tissues and organs.
Blood derivatives, such as platelet lysate (PL), have attracted great attention as an inexpensive milieu of bioactive molecules (e.g., growth factors, cytokines), self-assembling scaffolding proteins (e.g., fibrinogen, fibronectin, vitronectin), and antimicrobial peptides (e.g., platelet factor-4) capable of enhancing angiogenesis, stem cell recruitment, and tissue regeneration.[14, 15] To leverage these key biological cues, injectable PL-based nanocomposite fibrillar hydrogels were developed by triggering the natural in-situ clotting cascade in the presence of surface functionalized cellulose nanocrystals (CNC).[16] These hydrogels self-supported serum free culture of encapsulated stem cells, which showed excellent adhesion, fast proliferation and production of their own ECM, mainly due to the hydrogel physicochemical cues and intrinsic biological properties, i.e. These hydrogels self-supported serum free culture of encapsulated stem cells, which showed excellent adhesion, fast proliferation and production of their own ECM, mainly due to the hydrogel physicochemical cues and intrinsic biological properties, i.e. presence of adhesion motifs in the fibrillar proteins and PL bioactive molecules. Here, we exploit this concept to develop and test a humanbased bioink (HUink) for the freeform biofabrication of bioactive and biologically-relevant 3D structures. This advanced bioink enables the bottom-up biofabrication across multiple length scales into complex nano-to-macroscopic 3D fibrillar objects. Remarkably, human adipose-derived stem cells (hASCs) encapsulated in HUink present high cell viability after printing, while showing an exceptionally fast spreading, growth and synthesis of new ECM within the 3D construct over time in serum free culture conditions. Such physical and biofunctional properties demonstrate that HUink is a powerful bioink to print dynamic and personalized 3D living constructs that can be maturated in vitro in xeno-free conditions.

\section{Results and discussion}

\subsection{Bioprinting concept and characterization of the nanocomposite bioink}

HUink is obtained using a simple double barrel injection system filled with PL in one compartment and with thrombin, calcium and aldehyde-functionalized CNC (1.22-2.88 wt.\%) in the other (figure 1(a) and figure S1, SM). We first optimized the bioink precursors to obtain adequate viscosity and polymerization kinetics that allow for extrusion at low printing pressures (i.e. shear-thinning behaviour) and simultaneously leading to a self-assembled and crosslinked fibrillar matrix (figure 2(a) and (b)) by the activation of the fibrin polymerization with thrombin and calcium and the formation of Schiff base bonds between adehyde-CNC and amine groups of PL proteins (figure 1(a)).[16] PL was produced by the well-stablished method of repeated freeze/thaw cycles of platelet concentrates, which promotes 
platelet disruption and the release of the bioactive protein content.[14] To increase the protein content in the nanocomposite bioink, the initial PL was concentrated by reverse osmosis. The resulting solutions had a protein content of 67 and $160 \mathrm{mg} \mathrm{mL}^{-1}$, respectively, a process that had no significant impact on its viscosity (figure 2(a)). Among the tested formulations, we selected the conditions that result in printed structures with mechanical integrity and stability (i.e. PL $160 \mathrm{mg} \mathrm{mL}^{-1}$ and CNC 2.88 wt.\%) (figure S2, SM). Our bioprinting system was implemented in a broadly accessible 3D printing technology, which includes a commercially available Creatr 3D printer (Leapfrog), standard open source 3D printing software, and a modified stepper motor-driven thermoplastic extruder adapted to drive the plunger of $1.5 \mathrm{~mL}$ double barrel syringes $(1: 1)$ coupled with a nozzle equipped with a static mixer (figure 1(a)). Although HUink was easily extrudable through thin nozzles $(150-250 \mu \mathrm{m})$, its low viscosity and gelation kinetics do not allow building layer-bylayer 3D objects (figure 2(a) and (b)). Interestingly, recent works have shown that suspended manufacturing techniques allow for fabrication of 3D freeform hydrogel constructs with outstanding complexity and with minimal dependence on hydrogel rheological properties.[17-19] Following this concept, we used an agarose microparticles support matrix,[17] which behaves as a viscoelastic fluid and can selfheal after nozzle movement (figure 2(c) and (d)), as a 3D writing paper for HUink bioprinting. Simultaneously, the support matrix keeps our bioink in the printed position of the 3D space with minimal diffusion. Additionally, it enables the complete self-polymerization of HUink filaments while integrating the multiple printed layers by covalent crosslinking at crossing interfaces, resulting in high-fidelity constructs. Once the hydrogel crosslinking process is complete (reaches the steady state after $\sim 1$ hour, at $37^{\circ} \mathrm{C}$; figure 2 (b)), agarose can easily be washed out using PBS or culture media (figure 1(b) and Movie S1, SM).

\subsection{HUink printability and hierarchical structure}

The printability of the developed bioink was assessed by printing single filaments into the support matrix using different printing parameters. In order to observe the structure of the 3D printed hydrogel using confocal microscopy, fluorescent-labelled fibrinogen was added to PL. The diameter of the printed filaments $(102.3 \pm 21$ to $443.3 \pm 71.9 \mu \mathrm{m})$ could be controlled depending on the printing speed ( 5 to $30 \mathrm{~mm} \mathrm{~s}$ ${ }^{1}$ ) and nozzle size (25G to $28 \mathrm{G}$, corresponding to 250 to 150 $\mu \mathrm{m}$ internal diameter) (figure 3(a) and (b), and S4, SM). Among tested conditions, the filaments printed with 27-gauge needle and at $5 \mathrm{~mm} \mathrm{~s}^{-1}$ showed the highest fidelity and processability (figure 3(a) and (b), and S4, SM), and were therefore applied in the subsequent bioprinting assays. We could continuously print simple rectilinear lines in one axis, as well as patterns in two degrees of freedom with a rapid hydrogel deposition and minimal diffusion (figure 3(c)). Moreover, we were able to print in the $3 \mathrm{D}$ space constructs with multiple layers, e.g. a thick $(5 \mathrm{~mm})$ square lattice structure (figure 3(d)). HUink enabled the biofabrication of interconnected hierarchical fibrillar architectures spanning multiple length scales, starting from the nano self-assembled fibrin $\backslash \mathrm{CNC}$ fibrils $(52.7 \pm 11.5 \mathrm{~nm})$ bundled into fibres $(260.3 \pm 102.1 \mathrm{~nm})$ up to the macro infill patterns (figure 3(e)). By recapitulating the hierarchical and topographical architecture of the native fibrillar ECM, our system will certainly benefit the biological outcomes of bioprinted tissues and organs when compared to other typical polymer-based hydrogel bioinks.[20]

To show the versatility of HUink, we printed a variety of structures, including complex and large aspect ratio 3D objects, with different geometries and infill patterns (figure 4). These large-scale structures showed a high shape and mechanical integrity due to the fibrin self-assembly process that induced covalent fusion between deposited filaments, avoiding the common delamination between layers observed in $3 \mathrm{D}$ printed hydrogels using polymers that crosslink right after extrusion (Movie S2 and S3, SM).[21] Moreover, leveraging on the printing technology using supporting materials, the $3 \mathrm{D}$ printing process is not limited to standard layer-by-layer self-supported constructs and allows freeform fabrication according to the intended computer-aided design (Movie S4, SM). To demonstrate the freeform fabrication potential of the system, we printed a freestanding convex hemisphere with a radius of $\sim 12 \mathrm{~mm}$ that can maintain the complex printed geometry after removal of the supporting bath (figure 4(b)). Next, to show the construction of biologically-relevant structures, we printed a scaled-down human femur reconstructed from medical images (figure 4(d)) and a vasculature-like network from a leaf model (figure 4(e)). The intrinsic self-assembly and hierarchical ECM-mimetic network of HUink combined with the precise control over structure and material deposition provided by the supporting matrix, demonstrate its potential for the biofabrication of personalized human-size constructs.

\subsection{Bioprinting performance and biofunctionality of HUink}

The bioprinting and biological performance of the bioink was tested using human adipose tissue-derived stem cells (hASCs). These cells are harvested from adipose tissue in large numbers and have been studied as an excellent and widely available multipotent cell source for different tissue engineering applications.[22, 23] To demonstrate the outstanding HUink biofunctionality, we compared it with alginate-based and gelatin-methacrylate (GelMA) bioinks, two widely used hydrogel material in this field due to their convenient crosslinking mechanisms based on the use of calcium ions or UV light, respectively.[18, 24-26] Furthermore, to show that HUink can self-support the culture 
of the bioprinted constructs, all cellular assays were performed without any type of serum supplementation. All the produced bioinks laden with hASCs were printed using the same conditions. Shortly after extrusion ( 2 hours), cells in HUink showed significantly higher viability $(>90 \%)$ than in alginate constructs (> 60\%) and similar to GelMA (> 80\%) (figure 5(a) and (b)). These results confirm that the favourable rheological properties of HUink precursors (low viscosity of PL and marked shear-thinning behaviour of $\mathrm{CNC}$ ) decrease the shear stress exerted over the cells in the nozzle during extrusion in comparison to the alginate-based bioink (32 and $1161 \mathrm{~Pa}$, respectively), similar to the results obtained using GelMA (4 $\mathrm{Pa}$ ). The shear stress, which is known to affect the short and long-term cell signalling and behaviour, [25, 27, 28] exerted by HUink in combination with the cell-friendly printing process had a positive impact on post-printing cell viability. Notably, after 3 days in culture media hASCs already showed considerable cell spreading and spindle-like morphology whereas in alginate, cells maintained their original nonproliferative round shape, as would be expected from a hydrogel matrix without cell adhesion motifs. Moreover, although containing cell-binding motifs, cells in GelMA bioink also showed a similar round shape morphology observed in cells encapsulated in the alginate-based bioink. Unlike these commonly used polymer matrices, the enriched bioactive milieu of HUink contains key temporary ECM proteins (e.g. fibrin, fibronectin and vitronectin) with native cell binding motifs, and growth factors with strong mitogenic action (e.g. platelet-derived growth factor and fibroblast growth factor 2) that favour cell adhesion and fast proliferation, respectively.[14, 29] This is reflected in the significantly higher metabolic activity of cells in HUink compared to the other two bioink materials (figure 5(c)). In addition, although in this study pooled PL batches were prepared in house, the increasing trend on the use of standardized clinical grade human PL as xeno-free alternative to animal-derived serum in cell culture, $[30,31]$ represents an advantage over similar materials such as ECM bioinks (e.g. GelMA or decellurarised matrix),[11,32] which are typically of xenogenic origin, a significant barrier for clinical translation, and present high variability on the biochemical, topological and viscoelastic properties depending on the decellularization protocol. This further strengthens HUink concept, not only for research purposes, but also in terms of compliance with good manufacturing practices and clinical relevance.

After 9 days of culture, a high-density cellular network was formed independently of the infill pattern $(50 \%$ or $100 \%)$ (figure 6(a)), demonstrating that HUink promotes an unusual fast cell proliferation and cell-to-cell contact that contributes towards construct cellular densification. To further prove HUink superior bioactivity, GelMA constructs were printed and cultured in similar conditions and, as demonstrated in figure 6 (a), they show lower cellularity and cell spreading, in agreement with their comparatively lower cell metabolic activity (figure 5(c)). This unprecedented bioactive properties and ECM mimetic porous network represent a clear advantage over current biofabrication strategies, which typically depend on the use of perfusable channels or low infill patterns to minimize the diffusion limits of nutrients, metabolite and waste to sustain cell survival.[19, 33, 34] This was confirmed by comparing the differences between the diffusion of a model molecule through HUink and the alginate-based bioink (figure S3, SM). Remarkably, hASCs encapsulated in HUink deposited considerable amounts of collagen type I after just 9 days of culture, showing that cells are able to remodel the hydrogel network and replace it by their own ECM (figure 6(b)), contributing to the structural consolidation and integrity of the maturated constructs (figure 6(d) and S5, SM). To support this, we measured the shape factor of the 3D bioprinted constructs. These presented considerable shape fidelity to the 3D model right after printing (shape factor at day $0=1.1$ ) although they show some contraction after 1 day (shape factor at day $1=0.8$ ) but their dimensions and printed shape are well preserved through the following 9 days of culture (shape factor at day $9=0.8$ ), closely maintaining the height of the original 3D model $(0.26$ vs $0.25 \mathrm{~mm}$, respectively). Even though these constructs were bioprinted with $50 \%$ infilling, their shape integrity over culture time follows a similar trend to the observed for casted PL-CNC hydrogels with comparable PL:CNC proportion and cell content evaluated in our previous work. [16] On the other hand, cells in GelMA secreted minor amounts of collagen type I during this time period (figure 6(b)), even though the constructs remained with a high shape integrity (shape factor at day $9=1.1$ ) after 9 days of culture (figure S6, SM), reflecting the low remodelling of this matrix by cells cultured under the serum-free conditions. Furthermore, hASCs labelled with two different cell trackers were printed in discrete locations of the $3 \mathrm{D}$ space, providing proof-of-concept for the potential of the system to recreate patterns of heterocellular tissues (figure 6(c)). These results demonstrate that HUink not only possess outstanding biofunctional properties that can mimic the dynamics, composition and structure of native ECM and promote cell-cell communication, but collectively also results in the biofabrication of 3D living constructs, which are mechanically stabilized by a fast and newly deposited cell secreted matrix.

\section{Conclusions}

We have developed a unique bioactive human-based bioink that can be successfully used with the suspended manufacturing technology to biofabricate complex 3D freeform structures. This combined bioprinting platform has the capacity to print diverse freestanding constructs with hierarchical fibrillar architecture, high resolution, structural 
integrity and biocompatibility. The bioactivity of HUink scaffolding proteins and soluble factors, the low diffusional limitations provided by the fibrillar matrix and their susceptibility for cell remodelling can remarkably boost cell proliferation and colonization of $3 \mathrm{D}$ printed structures in culture conditions without any type of serum supplementation. The HUink concept is further supported by the increasing standardised production of clinical grade human PL as a powerful alternative to animal-origin serum for human mesenchymal stem cell expansion in future cell factories. Therefore, HUink unleashes many potential strategies to fabricate complex and personalized living constructs that can faithfully mimic tissue anatomy, which combined with straightforward bioprinting strategies and xeno-free culture conditions, supports its potential both as a singular platform for in vitro 3D studies as well as for translation from benchto-bedside.

\section{Experimental methods and materials}

\subsection{Platelet lysate preparation}

Platelet concentrates were obtained from healthy human blood donors provided by Serviço de Imuno-Hemoterapia of Centro Hospitalar de S. João, EPE (Porto, Portugal). The procedures were approved by the University of Minho Ethics Committee and all the platelet products were biologically qualified according to the Portuguese legislation. Platelet count was performed at HSJ and concentrates with a platelet count below 1 million platelet $\mu \mathrm{L}^{-1}$ were rejected. PL were prepared according to a previously established protocol.[35] Briefly, the pooled platelet concentrate samples (12 donors) were subject to three repeated temperature cycles (frozen with liquid nitrogen at $-196{ }^{\circ} \mathrm{C}$ and heated at $37{ }^{\circ} \mathrm{C}$ water bath). Aliquots of $\mathrm{PL}$ were stored at $-80^{\circ} \mathrm{C}$. Prior to use, the lysate was centrifuged at $4000 \mathrm{G}$ for 5 minutes and filtered through a $0.45 \mu \mathrm{m}$ pore filter to remove the platelet membrane fragments. For the concentration of PL, a reverse osmosis procedure using benzoylated cellulose dialysis membranes (2000 Da NMWCO, Sigma-Aldrich, USA) against poly(ethylene glycol) (average MW 20,000 kDa, SigmaAldrich, USA) was performed for $6 \mathrm{~h}$ at $4^{\circ} \mathrm{C}$.

\subsection{Aldehyde-CNC production}

CNC were extracted from microcrystalline cellulose (MCC) powder (Sigma-Aldrich, USA) following the typical sulfuric acid hydrolysis according to Bondeson with minor modifications.[36] Concentrated sulfuric acid (95-98\% from Sigma-Aldrich, USA) was added dropwise up to a final concentration of $64 \mathrm{wt} . \%$ and the obtained suspension was heated to $44{ }^{\circ} \mathrm{C}$ for $2 \mathrm{~h}$. The final working suspension of modified CNC was collected and stored at $4{ }^{\circ} \mathrm{C}$. Aldehyde $\mathrm{CNC}$ (a-CNC) were produced by sodium periodate $\left(\mathrm{NaIO}_{4}\right)$ oxidation.[37] In a typical experiment, $\mathrm{NaIO}_{4}$ (Sigma-

Aldrich, USA) was added to $\mathrm{CNC}$ aqueous suspension (1.5 wt.\%) in a 1:1 molar ratio (NaIO4:CNC) for 12 hours preventing. The desired concentration of the working suspension was adjusted by concentrating it against poly(ethylene glycol) (average MW 20,000 kDa, SigmaAldrich, USA) using benzoylated cellulose dialysis membranes (2000 Da NMWCO, Sigma-Aldrich, USA). a$\mathrm{CNC}$ were imaged using atomic force microscopy. Drops of the diluted CNC suspension (0.0015 wt.\%) were deposited on freshly cleaved and carefully washed mica discs $(9.9 \mathrm{~mm}$ diam. 0.27 thick). The suspension was left to adsorb for 15 minutes and the excess liquid was removed. The disc was washed two times with ultra-pure water and allowed to dry overnight. The samples were imaged in tapping mode with a MultiMode AFM (Bruker, USA) and the particle size distribution was determined with Gwyddion software $(n=50)$.

\subsection{HUink preparation}

Stock solutions of PL and a-CNC were produced and fully characterized as previously described. Double-barrel Lsystem syringes (1:1 ratio from Medmix, Switzerland) with a customized static mixer tip of $3 \mathrm{~mm}$ diameter and $6 \mathrm{~mm}$ height (102915, Medmix, Switzerland) were used to extrude the bioink. Barrel A was filled with PL (67-160 mg mL-1 of total dry mass). Barrel B contained thrombin from human plasma (5 $\mathrm{U} \mathrm{mL}^{-1}$, Sigma-Aldrich, USA), $\mathrm{CaCl}_{2}(10 \mathrm{mM}$, SigmaAldrich, USA) and a-CNC water dispersion (0-2.88 wt.\%). Concentrated PL (160 $\left.\mathrm{mg} \mathrm{mL}^{-1}\right)$ and a-CNC at approximately 18 wt. \% of PL total protein content (2.88 wt.\%) were used to produce HUink, unless otherwise stated. Based on the typical fibrinogen concentration range in $\mathrm{PL}\left(454 \pm 75 \mu \mathrm{g} \mathrm{mL}^{-1}\right)^{4} 21$ $\mu \mathrm{g}$ of Alexa Fluor ${ }^{\circledR} 488$ conjugated fibrinogen from human plasma (Thermo Fisher Scientific, USA) were added to each $1 \mathrm{~mL}$ of PL solution (barrel A) for visualization of the fibrin network using confocal microscopy.

\subsection{Alginate and gelatin-methacrylate bioinks}

A solution of $4 \%(\mathrm{w} / \mathrm{v})$ sodium alginate and $0.4 \%(\mathrm{w} / \mathrm{v})$ hyaluronic acid (MW 200,000, Lifecore Biomedical, USA) was prepared in PBS and stirred at $20^{\circ} \mathrm{C}$ for $48 \mathrm{~h}$, as previously described. [18]

GelMA was synthesized by reaction of gelatin (type-A, porcine skin, Sigma-Aldrich) with methacrylic anhydride (Sigma-Aldrich), as previously decribed.[38] The final bioink consisted in a solution of $10 \%(\mathrm{w} / \mathrm{v})$ GelMA and $0.5 \%$ Irgacure 2959 (Sigma-Aldrich) dissolved in PBS. Right after printing, constructs were photopolymerized by exposure to ultraviolet light $\left(320-500 \mathrm{~nm}, 1.2 \mathrm{~mW} \mathrm{~cm} \mathrm{~cm}^{-2}\right)$ for $60 \mathrm{~s}$ (Omnicure S2000).

\subsection{Support matrix preparation}


The support fluid gel was prepared as described by Moxon et al.[17] with slight modifications. A 0.5 wt.\% agarose (SeaKem, Lonza, USA) solution containing $11 \mathrm{mM} \mathrm{CaCl}_{2}$ (Sigma-Aldrich, USA) was autoclaved and cooled down to 20 ${ }^{\circ} \mathrm{C}$ under constant shear using a magnetic stirrer rotating at 700 rpm in order to form agarose microparticles.

\subsection{D printing of complex constructs using HUink}

All 3D printing was performed using a Creatr DualExtruder (Leapfrog, The Netherlands). One thermoplastic extruder was removed from the $x y$-axis carriage and the stepper motor was used to drive the plunger of a customized double-barrel syringe extruder. The custom syringe extruder was printed in acrylonitrile butadiene styrene (Leapfrog, The Netherlands) using the thermoplastic extruder of the same printer. 3D models were created using Autodesk Inventor (Adobe, USA) and exported to STL files. The file of the human femur was downloaded from NIH 3D Print Exchange and the leaf vasculature was downloaded from Thingiverse under creative commons licensing by Alajaz. The STL files were opened in Repetier-Host and sliced into 200- $\mu$ m-thick layers by Sli3r software to generate the G-code for $3 \mathrm{D}$ printing. The printing was done at room temperature $\left(20^{\circ} \mathrm{C}\right)$ at a speed of $5 \mathrm{~mm} \mathrm{~s}^{-1}$, unless otherwise stated, and different infill patterns and densities were used. To print inside of the support matrix, a custom post-processing script was added to Slic $3 r$ to move the $z$-stage and avoid the collision of the syringe with the petri dishes. HUink precursors were loaded into a double-barrel syringe capped with 27-gauge stainless steel needles (Nordson EFD, USA), unless otherwise stated. A container large enough to hold the construct was loaded with agarose support matrix and placed at the printing stage. The extruder was manually controlled from the Repetier software to push the plunger to initiate the extrusion right before start printing to avoid clogging in the nozzle. Constructs were allowed to full crosslink for $2 \mathrm{~h}$ before gently replacing the support matrix for PBS or culture media.

\subsection{Scanning electron microscopy}

Printed hydrogels were fixed in $2.5 \%$ glutaraldehyde (Merck, Germany) for 30 minutes. After fixation, samples were solvent exchanged from water to ethanol (ethanol gradient from 25, 50, 75 and 99.9 vol\%) for 4 hours and then critical point dried with $\mathrm{CO}_{2}$. After immersion in liquid nitrogen, the samples were freeze-fractured to expose their inner structures and sputter coated $(30$ seconds at $20 \mathrm{~mA}$, Cressington) with gold prior to observation in a scanning electron microscope (JSM-6010LV, JEOL, Japan).

\subsection{Cell experiments}

hASCs were obtained from lipoaspirate samples of the abdominal region of patients undergoing plastic surgery after the signature of an informed consent, under the scope of protocols established with Hospital da Prelada (Porto, Portugal) with the approval of the Hospital and University of Minho Ethics Committee. The hASCs isolation and stemness characterization were performed as previously optimized and described.[16, 39] hASCs were loaded to PL solution at a density of $2 \times 10^{6}$ cells per $1 \mathrm{~mL}$ of PL $\left(1 \times 10^{6}\right.$ cells per $1 \mathrm{~mL}$ of final hydrogel). In the alginate-based bioink and GelMA bioinks, hASCs were loaded at a density of $1 \times 10^{6}$ cells per 1 $\mathrm{mL}$ of hydrogel. All cell culture assays of hASCs encapsulated in bioinks were performed using culture media without serum supplementation.

To assess the shape fidelity of the 3D bioprinted construcs over time, $1 \times 1 \times 0.25 \mathrm{~cm}$ square lattices with $50 \%$ infilling were printed. At 1, 2 and $9 \mathrm{~d}$ after printing, top-view pictures of the constructs were taken with a digital camera and its surface area was measured using ImageJ. The shape factor was calculated by dividing the area of the printed construct at each culture period by the area of the 3D model.

\subsection{Fluorescent staining}

At $2 \mathrm{~h}$ and $72 \mathrm{~h}$ after printing, cellular viability was assessed using Calcein AM (Thermo Fisher Scientific, USA) and propidium iodide (Thermo Fisher Scientific, USA) to stain live and dead cells, respectively. After $9 \mathrm{~d}$ of culture, hydrogels were washed with PBS and then fixed in $10 \%$ formalin (Thermo Fisher Scientific, USA) for $15 \mathrm{~min}$ at RT and permeabilized using 0.2 vol.\% Triton-X100 (SigmaAldrich, USA). After washing, samples were incubated with $1: 200 \mathrm{v} / \mathrm{v}$ rhodamine-conjugated phalloidin (Sigma-Aldrich, USA) for 10 minutes and $1: 1000 \mathrm{v} / \mathrm{v} \mathrm{4}$, 6-diamidino- 2phenylindole (DAPI, Sigma-Aldrich, USA) for 20 minutes (dilutions in PBS). For collagen type I staining, samples were blocked using $3 \mathrm{w} / \mathrm{v} . \%$ BSA and incubated with 1:500 v.\% solution of specific primary antibody (ab90395, Abcam, UK). Then, samples were incubated with 1:200 v/v Alexa Fluor® 488 conjugated secondary antibody (Thermo Fisher Scientific, USA). For some experiments, hASCs were labelled with Calcein AM or with cell tracker CM-DiI dyes, prior to bioprinting. The HUink constructs were observed under a confocal microscope TCS SP8 (Leica Microsystems, Germany).

\subsection{Metabolic activity}

Metabolic activity in the different bioinks was assessed at 1, 2 and $7 \mathrm{~d}$ using Alamar Blue assay (Bio-Rad, USA), following the manufacturer's instructions.

\subsection{Rheology}

A Kinexus Pro Rheometer (Malvern Instruments, United Kingdom) was used to measure the viscoelastic properties of the materials. Time-sweep tests were applied to study 
polymerization kinetics of HUink. Immediately after dispensing $320 \mu \mathrm{L}$ of extruded solution on the bottom plate, a $20 \mathrm{~mm}$ diameter top plate was lowered to a final gap of $1 \mathrm{~mm}$ (parallel plate set up). A thin film of mineral oil was poured over the exposed surface to reduce water evaporation. This time point was defined as the starting time $(\mathrm{t}=0)$. Time-sweep assays were performed at a strain amplitude of $1 \%$ and frequency of $1 \mathrm{~Hz}$, and storage modulus values were monitored continuously at 10 seconds intervals. In shear sweep tests a $40 \mathrm{~mm} 4^{\circ}$ cone and plate geometry was used. The solutions viscosity was measured in response to the increasing of shear rate from 0.001 to $100 \mathrm{~s}^{-1}$. The same rheological set-up was used to perform frequency-sweep tests in the agarose support matrix with slight modifications from Moxon's work.[17] In stress-weep tests, agarose microparticles were subjected to a shear stress of 0.1-100 Pa at a constant oscillatory frequency of $1 \mathrm{~Hz}$. All measurements were performed in triplicate.

The shear stress $(\tau(\mathrm{Pa}))$ was calculated using Equation (1), where $\eta$ (Pa s) is viscosity and $\dot{\gamma}\left(\mathrm{s}^{-1}\right)$ the shear rate in the nozzle during extrusion, obtained from Equation (2):

$$
\begin{aligned}
\tau & =\eta \dot{\gamma} \\
\dot{\gamma} & =\frac{3 n+1}{n} \frac{Q}{\pi R^{3}}
\end{aligned}
$$

where $Q\left(\mathrm{~m}^{3} \mathrm{~s}^{-1}\right)$ is the flow, $R(\mathrm{~m})$ the radius of the nozzle and $n$ is the flow behavior index derived from the slope of the shear stress to shear rate graph using the Power-Law equation from 1 to $100 \mathrm{~s}^{-1}$. [25, 40]

\subsection{Diffusion of molecules through the bioinks}

HUink and alginate-based bioink were produced as previously described and injected in the central chamber of a chemotaxis $\mu$-slide (Ibidi, Germany) and the side chambers of the slide were filled with $10 \mathrm{mM} \mathrm{CaCl}_{2}$ allowing the hydrogels crosslinking to proceed for $2 \mathrm{~h}$. Then, the left chamber was filled with $25 \mu \mathrm{g} \mathrm{mL}-1$ of $20 \mathrm{kDa}$ dextran-FITC (SigmaAldrich, USA) in PBS and the right chamber with PBS. Fluorescence microscopy (Axio Observer, Zeiss, Germany) with incubation to simulate cell culture conditions $\left(37^{\circ} \mathrm{C}, 5 \%\right.$ $\mathrm{CO}_{2}$ ) was used to observe the diffusion of dextran-FITC through the bioinks over time (images were acquired every 3 min for $2 \mathrm{~h}$ ). The mean fluorescence intensity on a selected region of interest (ROI) in the right chamber was plotted against time.

\section{Acknowledgements}

The authors thank Hospital da Prelada (Porto, Portugal) for providing adipose tissue samples and Hospital São João (Porto, Portugal) for providing platelet concentrates. Authors acknowledge the financial support from project NORTE-010145-FEDER-000021 supported by Norte Portugal Regional Operational Programme (NORTE 2020), under the
PORTUGAL 2020 Partnership Agreement, through the European Regional Development Fund (ERDF); the European Union Framework Programme for Research and Innovation HORIZON 2020, under the TEAMING Grant agreement No 739572 - The Discoveries CTR EU, Marie Skłodowska-Curie grant agreement No 706996 and European Research Council grant agreement No 726178; FCT/MCTES (Fundação para a Ciência e a Tecnologia/ Ministério da Ciência, Tecnologia, e Ensino Superior) and the Fundo Social Europeu através do Programa Operacional do Capital Humano (FSE/POCH) in the framework of $\mathrm{PhD}$ grant $\mathrm{PD} / 59 / 2013$ PD/BD/113807/2015 for BBM, Post-Doc grant SFRH/BPD/112459/2015 for RMD. The authors would like to thank Tram Le, Carlos Guimarães and Luca Gasperini for their support to acquire pictures and design the drawings. The authors would also like to thank Syeda Mahwish Bakht for the gelatin rhelogical data acquisiton.

\section{References}

[1] Santoro M, Navarro J and Fisher J P 2018 Micro- and Macrobioprinting: Current Trends in Tissue Modeling and Organ Fabrication Small Methods 2 1700318

[2] Moroni L, Burdick J A, Highley C, Lee S J, Morimoto Y, Takeuchi S and Yoo J J 2018 Biofabrication strategies for 3D in vitro models and regenerative medicine Nature Reviews Materials 3 21-37

[3] Chimene D, Lennox K K, Kaunas R R and Gaharwar A K 2016 Advanced Bioinks for 3D Printing: A Materials Science Perspective Ann. Biomed. Eng. 44 2090-102

[4] Malda J, Visser J, Melchels F P, Jüngst T, Hennink W E, Dhert W J A, Groll J and Hutmacher D W 2013 25th Anniversary Article: Engineering Hydrogels for Biofabrication Advanced Materials 25 5011-28

[5] Katja H, Shengmao L, Liesbeth T, Sandra Van V, Linxia G and Aleksandr O 2016 Bioink properties before, during and after 3D bioprinting Biofabrication 8032002

[6] Bittner S M, Guo J L and Mikos A G 2018 Spatiotemporal control of growth factors in threedimensional printed scaffolds Bioprinting e00032

[7] Lee K, Silva E A and Mooney D J 2011 Growth factor delivery-based tissue engineering: general approaches and a review of recent developments Journal of the Royal Society Interface 8 153-70

[8] Hynes R O 2009 The extracellular matrix: not just pretty fibrils Science (New York, N.Y.) 326 1216-9

[9] Vogel V 2018 Unraveling the Mechanobiology of Extracellular Matrix Annual review of physiology $\mathbf{8 0}$ 353-87

[10] Vining K H and Mooney D J 2017 Mechanical forces direct stem cell behaviour in development and regeneration Nature reviews. Molecular cell biology $18728-42$ 
[11] Choudhury D, Tun H W, Wang T and Naing M W 2018 Organ-Derived Decellularized Extracellular Matrix: A Game Changer for Bioink Manufacturing? Trends in biotechnology 36 787-805

[12] Hedegaard C L, Collin E C, Redondo-Gómez C, Nguyen L T H, Ng K W, Castrejón-Pita A A, Castrejón-Pita J R and Mata A 2018 Hydrodynamically Guided Hierarchical SelfAssembly of Peptide-Protein Bioinks Advanced Functional Materials 281703716

[13] Prince E and Kumacheva E 2019 Design and applications of man-made biomimetic fibrillar hydrogels Nature Reviews Materials

[14] Mendes B B, Gomez-Florit M, Babo P S, Domingues R M, Reis R L and Gomes M E 2018 Blood derivatives awaken in regenerative medicine strategies to modulate wound healing Advanced drug delivery reviews 129 376-93

[15] Faramarzi N, Yazdi I K, Nabavinia M, Gemma A, Fanelli A, Caizzone A, Ptaszek L M, Sinha I, Khademhosseini A, Ruskin J N and Tamayol A 2018 Patient-Specific Bioinks for 3D Bioprinting of Tissue Engineering Scaffolds Advanced Healthcare Materials 71701347

[16] Mendes B B, Gómez-Florit M, Pires R A, Domingues R M A, Reis R L and Gomes M E 2018 Human-based fibrillar nanocomposite hydrogels as bioinstructive matrices to tune stem cell behavior Nanoscale

[17] Moxon S R, Cooke M E, Cox S C, Snow M, Jeys L, Jones S W, Smith A M and Grover L M 2017 Suspended Manufacture of Biological Structures Advanced Materials 291605594

[18] Hinton T J, Jallerat Q, Palchesko R N, Park J H, Grodzicki M S, Shue H J, Ramadan M H, Hudson A R and Feinberg A W 2015 Three-dimensional printing of complex biological structures by freeform reversible embedding of suspended hydrogels Science advances 1 e 1500758

[19] Highley C B, Rodell C B and Burdick J A 2015 Direct 3D Printing of Shear-Thinning Hydrogels into Self-Healing Hydrogels Advanced Materials 27 5075-9

[20] Murphy S V and Atala A 2014 3D bioprinting of tissues and organs Nature Biotechnology 32773

[21] Sears N A, Seshadri D R, Dhavalikar P S and Cosgriff-Hernandez E 2016 A Review of ThreeDimensional Printing in Tissue Engineering Tissue Engineering Part B: Reviews 22 298-310

[22] Gimble J M, Katz A J and Bunnell B A 2007 Adipose-derived stem cells for regenerative medicine Circulation research 100 1249-60

[23] Rada T, Reis R L and Gomes M E 2009 Adipose Tissue-Derived Stem Cells and Their Application in Bone and Cartilage Tissue Engineering Tissue Engineering Part B: Reviews 15 113-25

[24] Jung-Seob L, Jung Min H, Jin Woo J, Jin-Hyung S, Jeong-Hoon O and Dong-Woo C 2014 3D printing of composite tissue with complex shape applied to ear regeneration Biofabrication 6024103

[25] Blaeser A, Duarte Campos D F, Puster U, Richtering W, Stevens M M and Fischer H 2016 Controlling Shear Stress in 3D Bioprinting is a Key Factor to Balance Printing Resolution and Stem Cell Integrity Adv Healthc Mater 5 326-33

[26] Cidonio G, Glinka M, Dawson J I and Oreffo R O C 2019 The cell in the ink: Improving biofabrication by printing stem cells for skeletal regenerative medicine Biomaterials 209 10-24

[27] Galie P A, Nguyen D-H T, Choi C K, Cohen D M, Janmey P A and Chen C S 2014 Fluid shear stress threshold regulates angiogenic sprouting Proceedings of the National Academy of Sciences 1117968

[28] Busch R, Strohbach A, Pennewitz M, Lorenz F, Bahls M, Busch M C and Felix S B 2015 Regulation of the endothelial apelin/APJ system by hemodynamic fluid flow Cellular Signalling $\mathbf{2 7}$ 1286-96

[29] Rosales A M and Anseth K S 2016 The design of reversible hydrogels to capture extracellular matrix dynamics Nature reviews. Materials 1

Becherucci V, Piccini L, Casamassima S, Bisin S, Gori V, Gentile F, Ceccantini R, De Rienzo E, Bindi B, Pavan P, Cunial V, Allegro E, Ermini S, Brugnolo F, Astori G and Bambi F 2018 Human platelet lysate in mesenchymal stromal cell expansion according to a GMP grade protocol: a cell factory experience Stem Cell Research \& Therapy 9124

[31] Saury C, Lardenois A, Schleder C, Leroux I, Lieubeau B, David L, Charrier M, Guével L, Viau S, Delorme B and Rouger K 2018 Human serum and platelet lysate are appropriate xeno-free alternatives for clinical-grade production of human MuStem cell batches Stem Cell Research \& Therapy 9128

[32] Hussey G S, Dziki J L and Badylak S F 2018 Extracellular matrix-based materials for regenerative medicine Nature Reviews Materials 3 159-73

[33] Kolesky D B, Homan K A, Skylar-Scott M A and Lewis J A 2016 Three-dimensional bioprinting of thick vascularized tissues Proceedings of the National Academy of Sciences of the United States of America 113 3179-84

[34] Miri A K, Khalilpour A, Cecen B, Maharjan S, Shin S R and Khademhosseini A 2018 Multiscale bioprinting of vascularized models Biomaterials

[35] Santo V E, Gomes M E, Mano J F and Reis R L 2012 Chitosan-chondroitin sulphate nanoparticles for controlled delivery of platelet lysates in bone regenerative medicine Journal of tissue engineering and regenerative medicine 6 47-59

[36] Bondeson D, Mathew A and Oksman K 2006 Optimization of the isolation of nanocrystals from microcrystalline celluloseby acid hydrolysis Cellulose 13171 
[37] Domingues R M A, Silva M, Gershovich P, Betta S, Babo P, Caridade S G, Mano J F, Motta A, Reis R L and Gomes M E 2015 Development of Injectable Hyaluronic Acid/Cellulose Nanocrystals Bionanocomposite Hydrogels for Tissue Engineering Applications Bioconjugate chemistry 26 1571-81

[38] Akbari M, Tamayol A, Laforte V, Annabi N, Hassani Najafabadi A, Khademhosseini A and Juncker D 2014 Composite Living Fibers for Creating Tissue Constructs Using Textile Techniques Adv. Funct. Mater. 24 4060-7

[39] Carvalho P P, Wu X, Yu G, Dias I R, Gomes M E, Reis R L and Gimble J M 2011 The effect of storage time on adipose-derived stem cell recovery from human lipoaspirates Cells, tissues, organs 194 494500

[40] Pourchet L J, Thepot A, Albouy M, Courtial E J, Boher A, Blum L J and Marquette C A 2017 Human Skin 3D Bioprinting Using Scaffold-Free Approach Advanced Healthcare Materials 61601101 
(a)

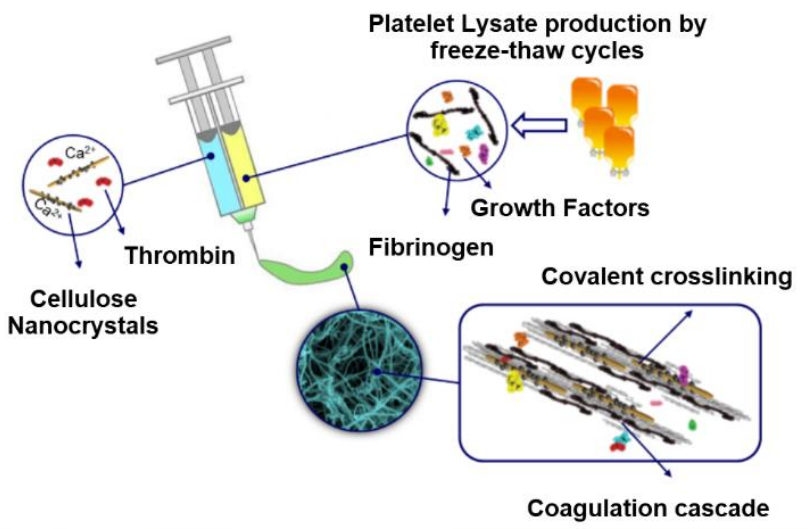

(b) HUink composition

i

ii

iii
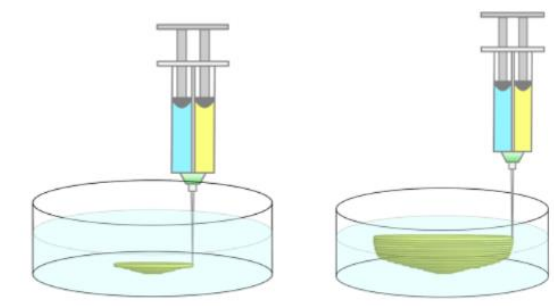

HUink printing process

Figure 1. HUink composition and bioprinting process. (a) Schematic illustration of HUink components and the crosslinking reaction. (b) Biofabrication process, namely HUink is extruded layer-by-layer into the agarose support bath (i), after the complete printing of the freeform construct and its full crosslinking (ii), the freeform 3D construct is removed from the support bath (iii). 
(a)

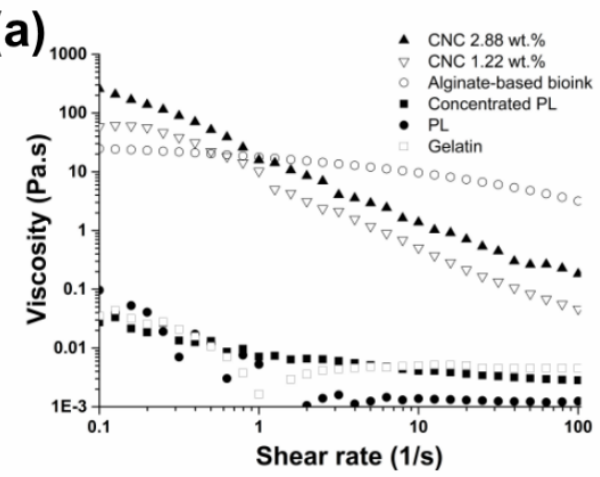

(c)

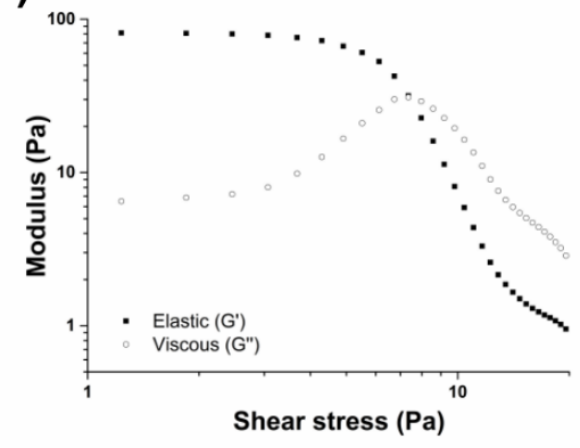

(b)

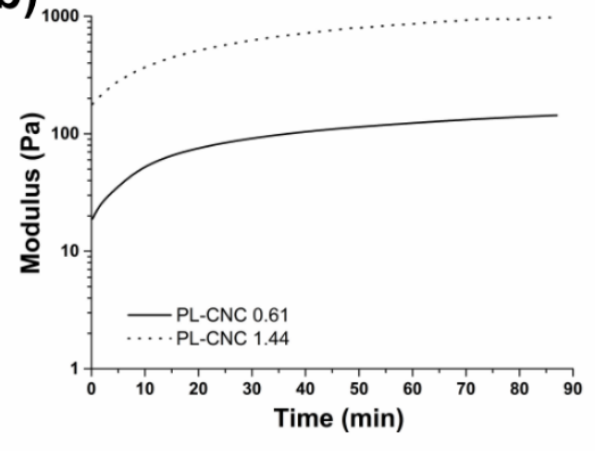

(d)

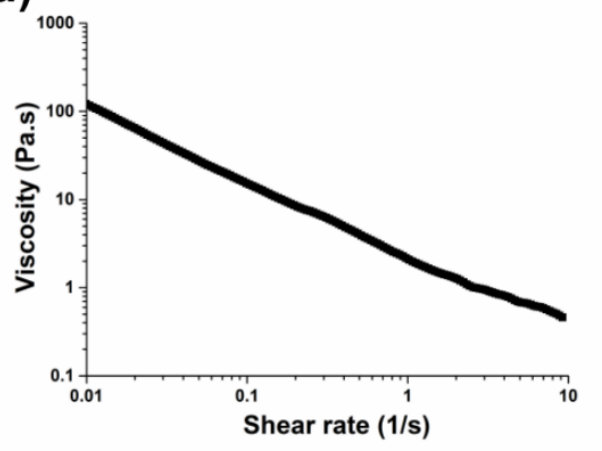

Figure 2. Rheological characterization. (a) Bioink precursor's viscosity in response to increasing shear rates from 0.1 to $100 \mathrm{~s}^{-1}$. (b) Polymerization of PL-CNC biomaterial with different precursors concentrations, PL-CNC 0.61 data obtained from [16]. Agarose microparticulate bath rheological properties, namely (c) elastic and viscous modulus in response to increasing shear stress and (c) viscosity in response to increasing shear rates. 
(a)

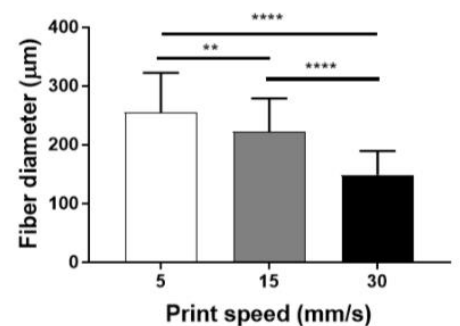

(c)

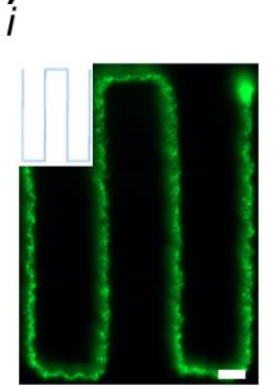

(e)

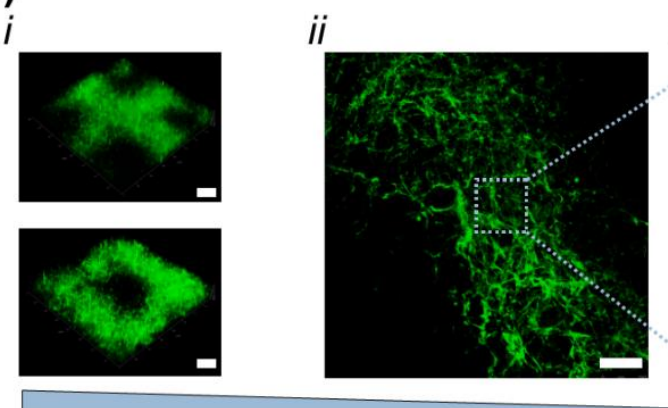

Macro

ii

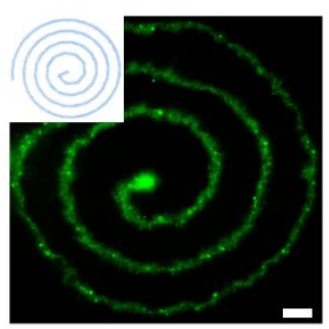

(b)

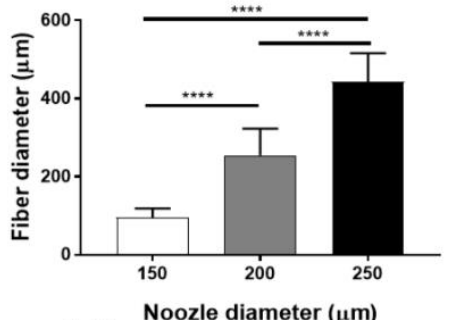

(d)

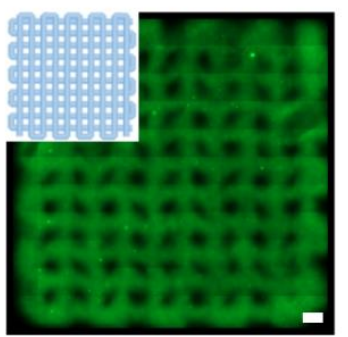

iii

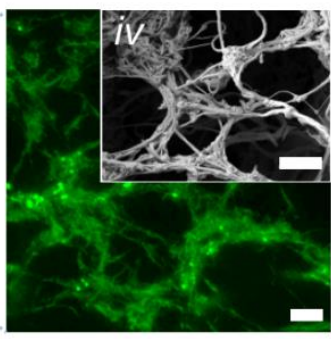

Nano

Figure 3. HUink bioprinting performance and fibrillar hierarchical structure. Filaments with different widths were printed into the agarose support bath (a) from a 27-gauge needle and varying the velocity and (b) with a velocity of $5 \mathrm{~mm} / \mathrm{s}$ and modifying the needle diameter. (c) HUink printed into a continuous filament (i) or a concentric circle (ii) 2D pattern. (d) Thick 3D square lattice construct (30 layers). (e) Hierarchical fibrous structure from the macroscale pattern (i) of micro filaments (ii) composed of selfassembled fibrin nanofibrils (iii-iv). Staining in (c-e): fibrin-AlexaFluor 488 (green). Scale bars in (c): $250 \mu \mathrm{m},(\mathrm{d}): 1 \mathrm{~mm}$, (e-i): $200 \mu \mathrm{m}$, (e-ii): $100 \mu \mathrm{m}$, (e-iii): $10 \mu \mathrm{m}$, (e-iv): $2 \mu \mathrm{m}$. Statistical differences evaluated by one-way ANOVA using Tukey's multiple comparisons: $* * \mathrm{P}<0.01$ and $* * * * \mathrm{P}<0.0001$. 


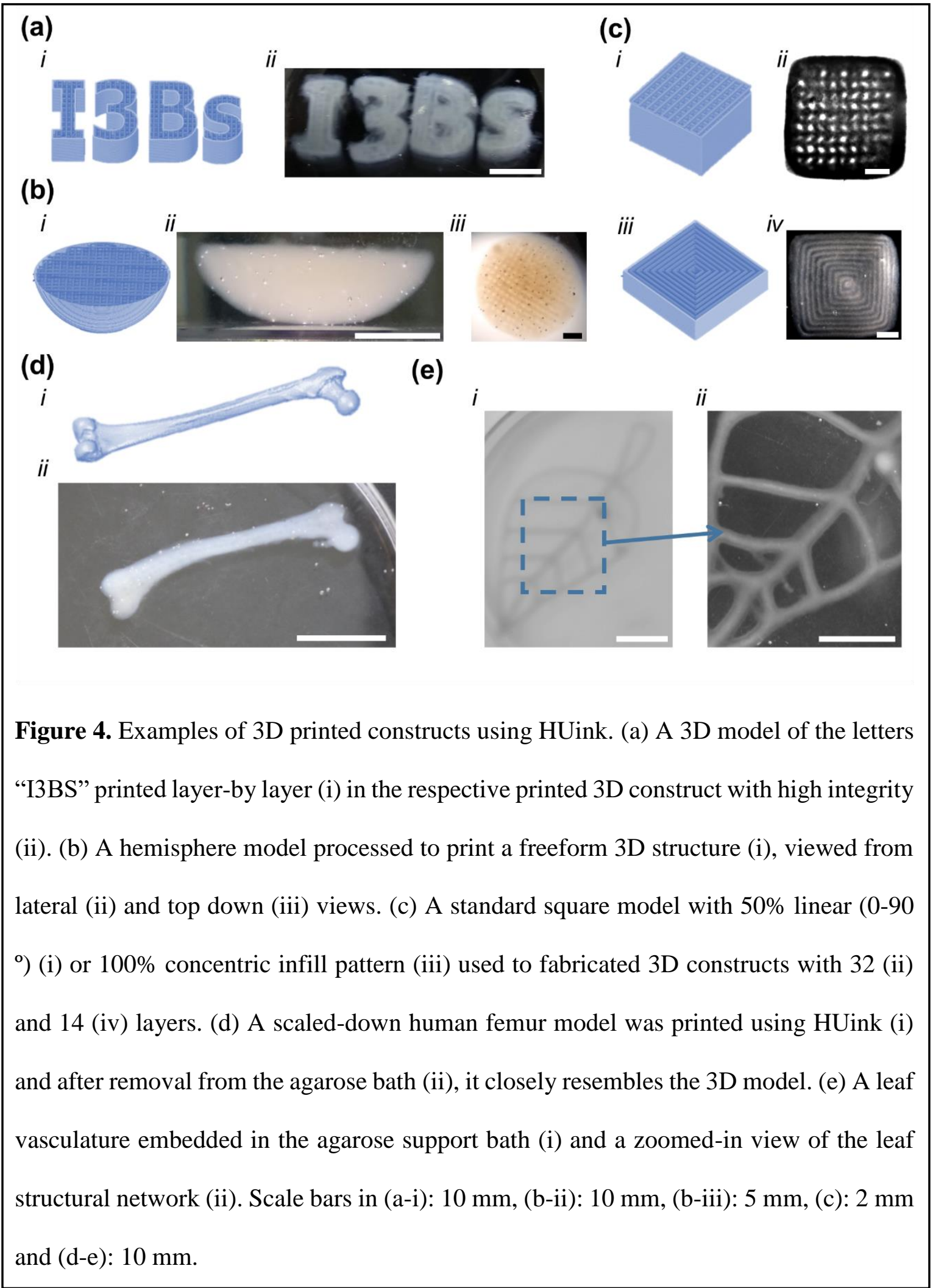



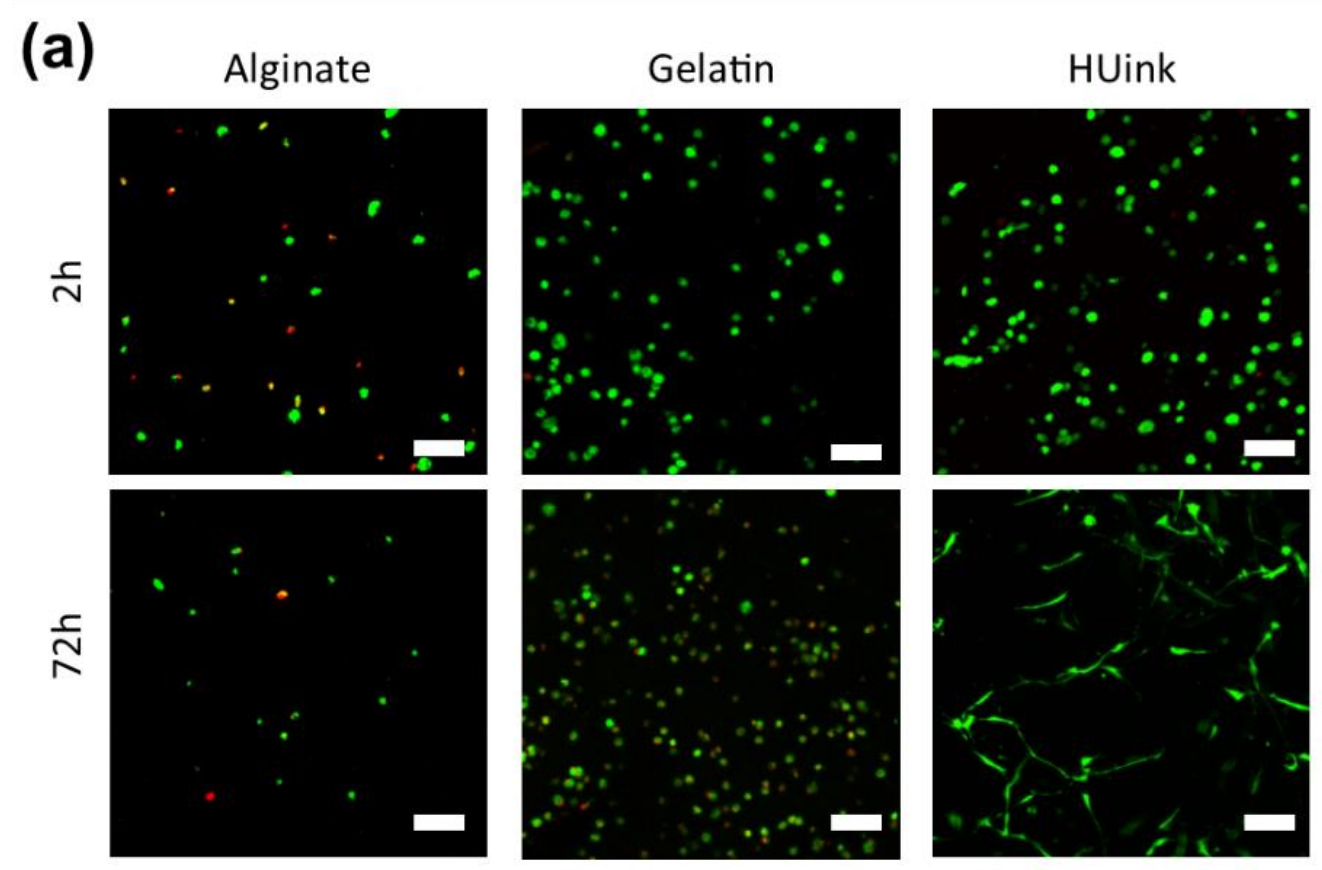

(b)
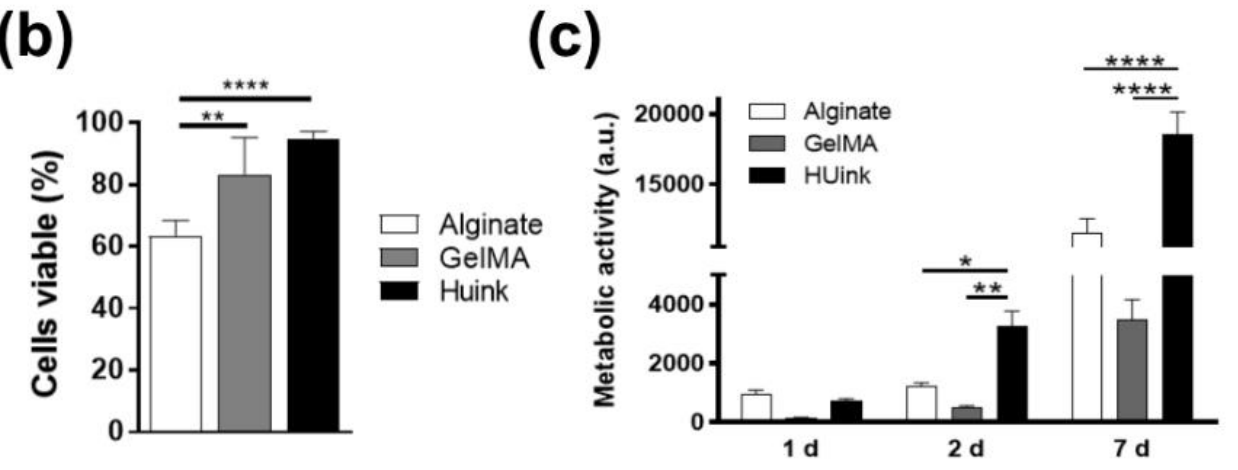

Figure 5. Biocompatibility and metabolic activity after bioprinting using hASCs. (a) Cell viability in alginate, GelMA and HUink 3D printed constructs with Live (green) / Dead (red) staining at 2 and 72 hours after printing. Scale bars $100 \mu \mathrm{m}$. (b) Measurement of viable cells (live cells from Live/Dead staining) 2 hours after the printing process $(n=3)$. (c) Metabolic activity of cells in the different bioinks over time. Statistical differences evaluated by (b) t-test: **** $\mathrm{P}<0.0001$; (c) one-way ANOVA using Tukey's multiple comparisons: $* \mathrm{P}<0.05, * * \mathrm{P}<0.01$ and $* * * * \mathrm{P}<0.0001$ 


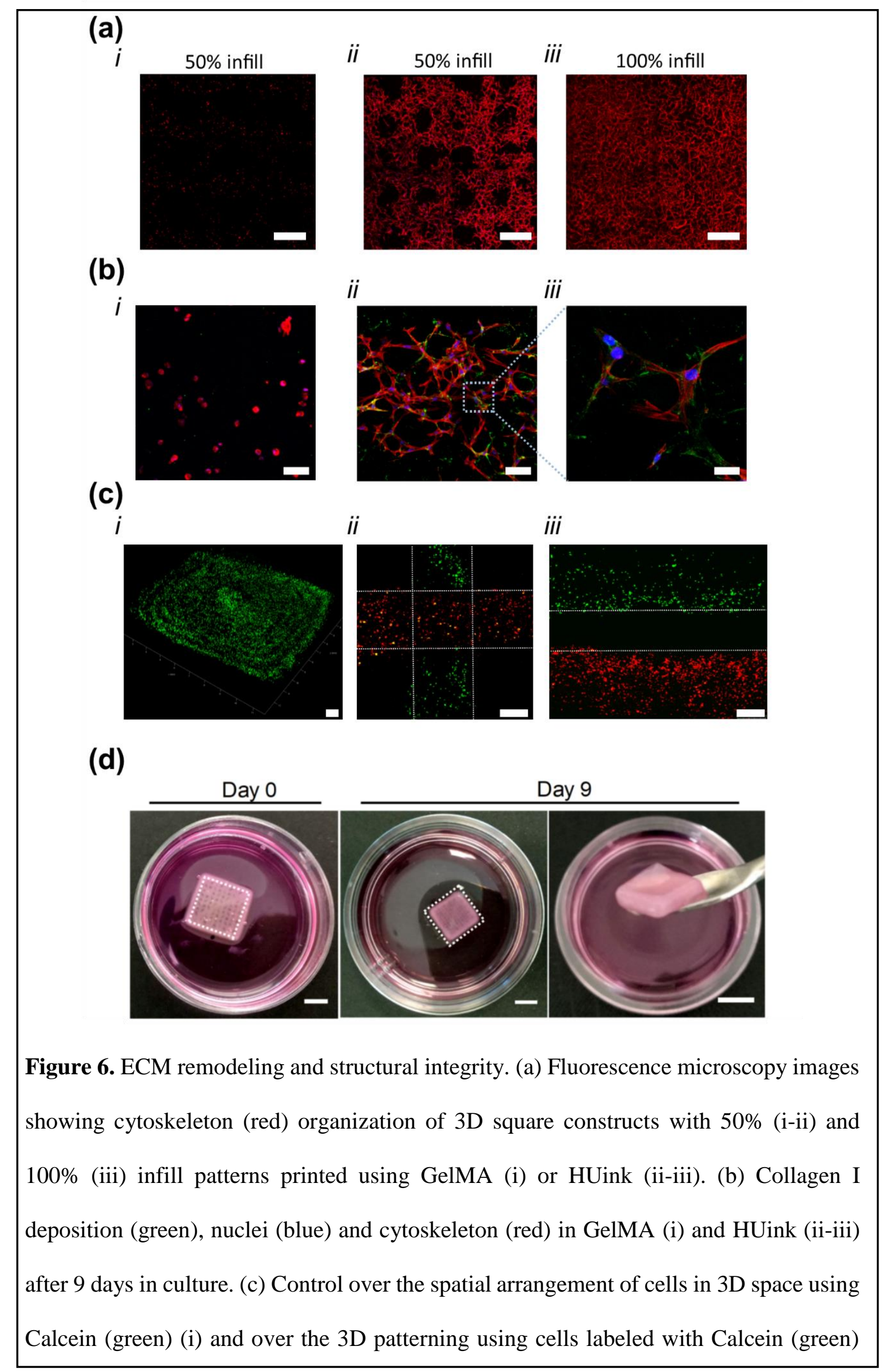


and CM-DiI (red) in perpendicular (ii) and parallel (iii) deposition. (d) Structural integrity of HUink constructs over culture time. Scale bars in (a): $500 \mu \mathrm{m}$, (b-i-ii): $75 \mu \mathrm{m}$, (b-iii): $25 \mu \mathrm{m}$, (c): $1 \mathrm{~mm}$ and (d): $0.5 \mathrm{~cm}$. The dotted square in (d) represents the size of the initial 3D model. 